\title{
A Study on the Mannich Reaction with 1,3-Indandione
}

\author{
E. M. Afsah, M. Hammouda*, and M. M. Khalifa \\ Chemistry Department, Faculty of Science, Mansoura University, Mansoura, A.R. Egypt \\ Z. Naturforsch. 45b, 1055-1058 (1990); received October 24, 1989 \\ Mannich Reaction, 1,3-Indandione
}

Attempted Mannich reaction with the title compound $\mathbf{1}$ using morpholine and formalin led to $\mathbf{2}$ or $\mathbf{4}$ according to the reaction conditions. Whereas, on using primary amines the diindeno [1,2-b:2', $1^{\prime}$-e]pyridine (9-11) were obtained, in addition to 2 -methylene-1,3-indandione (6), in case of 10 and 11.

However, the Mannich bases 13-15 were formed on treating 2-benzylidene-1,3-indandione with the appropriate secondary amine. The structures 2, 9-11 were confirmed via unambiguous syntheses.

A search of the literature showed that the only reported case of Mannich reaction with 1,3-indandione (1) failed [1]. However, a number of 2-aryland aralkyl-2-aminomethyl-1,3-indandiones with psychopharmacological and anticoagulant activity [2], and also the pharmaceutically acceptable oxazidione [3] "2-(morpholinomethyl)-2-phenyl-1,3indandione" were reported in the patent literature. Prompted by these reports and in continuation of our work in this area [4-8], we investigated the Mannich reaction of $\mathbf{1}$ under different conditions.

Treatment of $\mathbf{1}$ with morpholine and formalin in ethanol-acetic acid, gave a sole product which was identified as 2,2'-methylene-bis[1-(N-morpholino)-3-indenone] (2), and non of the expected Mannich base $\mathbf{3}$ was obtained. On the other hand, the same reaction in acetic acid medium afforded a single product which was free from nitrogen, and insoluble in most organic solvents. The structure $\mathbf{4}$ assigned to this product is based on its analytical and IR spectral data.

Formation of compound $\mathbf{2}$ instead of $\mathbf{3}$ may be rationalized on the basis of a mechanism which involves the formation of 2-methylene-1,3-indandione (6) and this will further react with carbanion 5 to give 2,2'-methylene-bis-1,3-indandione (7) (Scheme 2). Therefore, compound $\mathbf{2}$ is formed, rather than $\mathbf{3}$, which would be obtained if $\mathbf{6}$ is attacked by morpholine instead of $\mathbf{5}$. This is because morpholine is less nucleophilic than the carbanion $\mathbf{5}$, and will not attack $\mathbf{6}$ as fast as the carbanion

\footnotetext{
* Reprint requests to Dr. M. Hammouda.

Verlag der Zeitschrift für Naturforschung, D-7400 Tübingen 0932-0776/90/0700-1055/\$01.00/0
}

will. In order to confirm this mechanism and structure of $\mathbf{2}$, we prepared $\mathbf{7}$ [9] which was treated with morpholine to give $\mathbf{2}$ (Scheme 1).

The spiro compound $\mathbf{4}$ is presumably formed by a mechanism which involves the intramolecular cyclization of the prefounded species (Scheme 3).

Obviously, anion 7 is the main intermediate in the reaction sequence, and its formation is expected in line of the behaviour of dimedone [10], and our report on Mannich reaction with 5-phenylcyclohexan-1,3-dione [11].

On the other hand, when 1 was treated with methylamine and formalin, it gave 5-methyl$5 \mathrm{H}$-diindeno[1,2-b:2', 1'-e]pyridine-10,12(11 H)dione (9), the NMR spectrum of which showed signals at $\delta 2.50\left(\mathrm{~N}-\mathrm{CH}_{3}\right)$ and 3.65 cyclic $\left(-\mathrm{CH}_{2}-\right)$.

Similarly, compounds $\mathbf{1 0}$ and $\mathbf{1 1}$ were obtained on using benzylamine or ammonium acetate respectively. In its NMR spectrum compound $\mathbf{1 0}$ showed signals at $\delta 2.75\left(\mathrm{Ph}-\mathrm{CH}_{2}-\mathrm{N}\right)$ and 3.60 cyclic $\left(-\mathrm{CH}_{2}-\right)$, in addition, a singlet was observed at $\delta 4.90(2 \mathrm{H})$ attributable to a vinylic $\left(=\mathrm{CH}_{2}\right)$ protons, indicating the presence of 2-methylene-1,3-indandione (6) as a fleeting intermediate. This was also observed in case of 11. It is therefore reasonable to assume that $\mathbf{6}$ is one of the intermediates in the reaction sequence, and that compounds 9-11 were formed via a mechanism which is identical to that of Scheme 2. The final step in such mechanism is the amination of 7 with primary amines to give $\mathbf{9 - 1 1}$.

The unambiguous synthesis of compounds 9-11 by treating 7 [9] with the appropriate amine confirmed their structures. This method afforded a pure samples of $\mathbf{1 0}$ and $\mathbf{1 1}$, whereas, $\mathbf{9}$ was identical to that obtained from $\mathbf{1}$. 


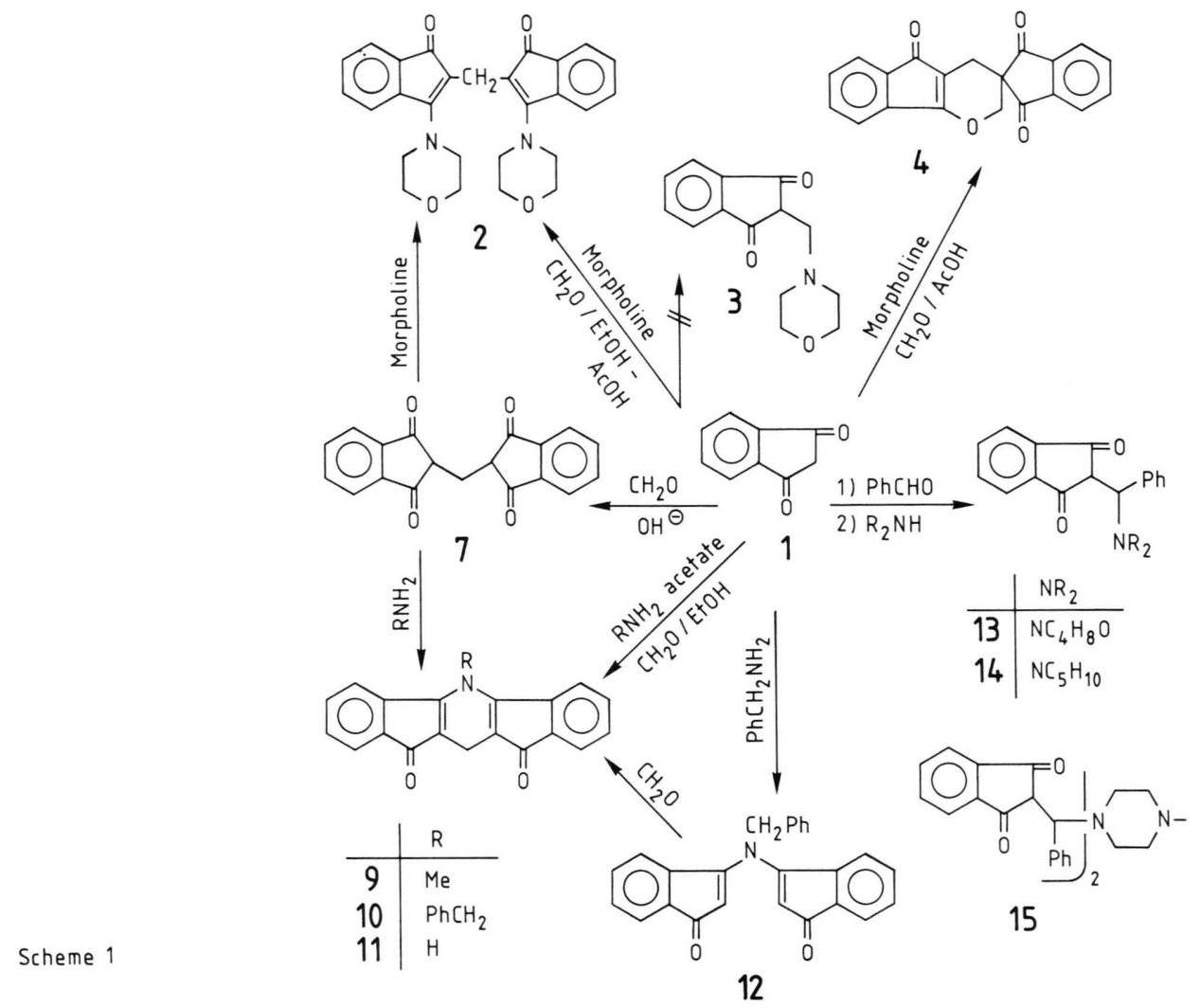

$1 \stackrel{\mathrm{R}_{2} \ddot{\mathrm{NH}}}{\longrightarrow} \stackrel{\mathrm{H}^{+}}{\longrightarrow} \stackrel{\mathrm{CH}_{2} \mathrm{O}}{\longrightarrow}$

5<smiles>C=C1C(=O)c2ccccc2C1=O</smiles>

Scheme 2
In an alternative route to the diindeno[1,2-b:2', $1^{\prime}$-e]pyridine ring system, we prepared N,N-di(3-indenon-1-yl)-benzylamine (12), which is a possible precursor to compound $\mathbf{1 0}$. Treatment of $\mathbf{1 2}$ with formalin afforded $\mathbf{1 0}$, such reaction is in line with the work of Greenhil [12] on the reactions of enaminones with aldehydes.

It appeared therefore, that formation of Mannich bases related to 1 can be achieved by treating a preformed 2-methylene-1,3-indandione (6) with amines. However, our attempts to prepare such

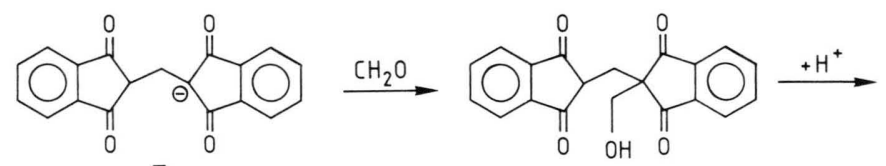

Carbonian of 7
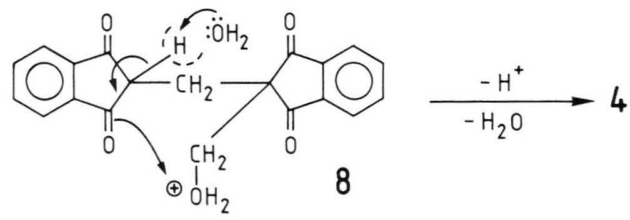
compound failed. Therefore, we prepared Mannich bases 13-15 by treatment of 2-benzylidene1,3-indandione [13] with morpholine, piperidine or piperazine, respectively. In this type of synthesis, the aminomethyl side chain must have an aryl fragment.

\section{Experimental}

Analytical data were determined by the Microanalytical Unit, Faculty of Science, Mansoura University. Infra-red spectra were recorded on $\mathrm{KBr}$ discs using a Unicam SP 2000 spectrophotometer. NMR spectra in $\mathrm{CDCl}_{3}$ solution were obtained on a Varian EM-360 $60 \mathrm{MHz}$ spectrometer with TMS as an internal standard. Melting points were uncorrected.

\section{2,2'-Methylene-bis [1-(N-morpholino)-3- indenone ] (2)}

(A) A solution of 1,3-indandione (1) $(0.01 \mathrm{~mol}$, $1.46 \mathrm{~g})$, formalin $(37 \%)(0.01 \mathrm{~mol}, 0.81 \mathrm{ml})$ and morpholine $(0.01 \mathrm{~mol}, 0.87 \mathrm{ml})$ in ethanol $(40 \mathrm{ml})$ and few drops of acetic acid, was kept at room temperature for $24 \mathrm{~h}$. The solid which separated was recrystallized from ethanol to give pink crystals, m.p. $175{ }^{\circ} \mathrm{C}$ in $(1.7 \mathrm{~g}) 75 \%$ yield. IR $1720(\mathrm{CO}$ of indenone) and $1370 \mathrm{~cm}^{-1}(\mathrm{C}-\mathrm{N}$ stretch.).

$$
\begin{array}{rrrr}
\mathrm{C}_{27} \mathrm{H}_{26} \mathrm{~N}_{2} \mathrm{O}_{4}(442.05) & & \\
\text { Calcd } & \mathrm{C} 73.28 & \mathrm{H} 5.92 & \text { N } 6.33, \\
\text { Found } & \mathrm{C} 73.04 & \mathrm{H} 5.66 & \text { N 6.37. }
\end{array}
$$

(B) A solution of 2,2'-methylene-bis-1,3-indandione (7) $(0.01 \mathrm{~mol}, 3.04 \mathrm{~g})$ and morpholine $(0.02 \mathrm{~mol}, 1.75 \mathrm{ml})$ in ethanol $(50 \mathrm{ml})$ and few drops of acetic acid, was kept at room temperature for $24 \mathrm{~h}$, then worked up as described above, m.p. $175^{\circ} \mathrm{C}$ in $(3.7 \mathrm{~g}) 84 \%$ yield (mixed m.p.).

\section{3,4-Dihydro-2 H-indeno[ 1,2-b]pyrano [3-spiro-2']-} indan- $1^{\prime}, 3^{\prime}, 5$-trione (4)

A solution of $1(0.01 \mathrm{~mol}, 1.46 \mathrm{~g})$, formalin $(37 \%) \quad(0.01 \mathrm{~mol}, \quad 0.81 \mathrm{ml})$ and morpholine
$(0.01 \mathrm{~mol}, 0.87 \mathrm{ml})$ or piperidine $(0.01 \mathrm{~mol}, 1 \mathrm{ml})$ in acetic acid $(30 \mathrm{ml})$ was heated on a steam bath for $2 \mathrm{~h}$. The solid product that obtained on cooling was collected, washed several times with hot alcohol to give 4 as white powder, m.p. $280{ }^{\circ} \mathrm{C}$ in $(1 \mathrm{~g})$ $62 \%$ yield.

$$
\begin{array}{lll}
\mathrm{C}_{20} \mathrm{H}_{12} \mathrm{O}_{4}(316.3) & \\
\text { Calcd } & \mathrm{C} 75.94 & \mathrm{H} 3.83, \\
\text { Found } \mathrm{C} 76.02 & \mathrm{H} 3.54 .
\end{array}
$$

IR 1730 (CO, indandione) and $1695 \mathrm{~cm}^{-1}(\mathrm{CO}$, indenone).

5-Methyl-5 H-diindeno[ 1,2-b:2', $1^{\prime}$-e]pyridine10,12(11 H)-dione (9), 5-benzyl-5 H-diindeno [ 1,2$b: 2^{\prime}, 1^{\prime}$-e Jpyridine-10,12(11 H)-dione $(\mathbf{1 0})$ and $5 \mathrm{H}$-diindeno $\left[1,2-b: 2^{\prime}, 1^{\prime}\right.$-e]pyridine-10,12(11 H)dione (11)

Procedure A: From 1,3-indandione: A solution of $1(0.01 \mathrm{~mol}, 1.46 \mathrm{~g})$; formalin $(37 \%)(0.01 \mathrm{~mol}$, $0.81 \mathrm{ml})$ and $(40 \%)$ methylamine $(0.01 \mathrm{~mol}$, $0.86 \mathrm{ml})$ or benzylamine $(0.01 \mathrm{~mol}, 1.1 \mathrm{ml})$ or ammonium acetate $(0.01 \mathrm{~mol}, 0.77 \mathrm{~g})$ in ethanol $(40 \mathrm{ml})$ and few drops of acetic acid, was kept overnight at room temperature. The reaction mixture was diluted with water $(50 \mathrm{ml})$, basified to $\mathrm{pH} 8$ by ammonia. The precipitated products were recrystallized from ethanol ( $c f$. Table I).

Procedure B: From 2,2'-methylene-bis-1,3-indandione: A solution of $7(10 \mathrm{mmol}, 3.04 \mathrm{~g})$ in glacial acetic acid $(10 \mathrm{ml}),(98 \%)$ methylamine $(12 \mathrm{mmol}, \quad 0.38 \mathrm{~g})$ or benzylamine $(12 \mathrm{mmol}$, $1.31 \mathrm{~g})$ or ammonium acetate $(12 \mathrm{mmol}, 0.93 \mathrm{~g})$ in acetic acid $(10 \mathrm{ml})$, was heated on a steam bath for $3 \mathrm{~h}$, left to cool, diluted with water. The solid obtained was recrystallized from ethanol to give $\mathbf{9 , 1 0}$ and 11 in $(1.95 \mathrm{~g}) 65 \%,(2.9 \mathrm{~g}) 77 \%$ and $(2.3 \mathrm{~g})$ $80 \%$ yield respectively.

Procedure $C$ : From N,N-di(3-indenon-1-yl)-

\begin{tabular}{|c|c|c|c|c|c|c|}
\hline Compound & Colour & $\begin{array}{l}\text { M.p. } \\
{\left[{ }^{\circ} \mathrm{C}\right]}\end{array}$ & $\begin{array}{l}\text { Yield } \\
{[\%]}\end{array}$ & $\begin{array}{l}\text { Formula } \\
\text { (mol. wt.) }\end{array}$ & $\begin{array}{l}\text { Analyses (\%) } \\
\text { C }\end{array}$ & $\begin{array}{l}\text { Found } \\
\text { (calcd) } \\
\mathrm{H}\end{array}$ \\
\hline 9 & brown & 185 & 70 & $\begin{array}{l}\mathrm{C}_{20} \mathrm{H}_{13} \mathrm{NO}_{2} \\
(299.3)\end{array}$ & $\begin{array}{c}79.98 \\
(80.25)\end{array}$ & $\begin{array}{c}4.02 \\
(4.37)\end{array}$ \\
\hline 10 & greenish & 238 & 82 & $\begin{array}{l}\mathrm{C}_{26} \mathrm{H}_{17} \mathrm{NO}_{2} \\
(375.4)\end{array}$ & $\begin{array}{c}83.00 \\
(83.18)\end{array}$ & $\begin{array}{c}4.35 \\
(4.56)\end{array}$ \\
\hline 11 & brown & 278 & 79 & $\begin{array}{l}\mathrm{C}_{19} \mathrm{H}_{11} \mathrm{NO}_{2} \\
(285.3)\end{array}$ & $\begin{array}{c}80.02 \\
(79.98)\end{array}$ & $\begin{array}{c}3.63 \\
(3.89)\end{array}$ \\
\hline
\end{tabular}
benzylamine (12): A solution of $12(0.01 \mathrm{~mol}$, $3.63 \mathrm{~g})$, formalin $(37 \%)(0.01 \mathrm{~mol}, 0.81 \mathrm{ml})$ in ethanol $(40 \mathrm{ml})$, was kept overnight at room temperature. The separated product was recrystallized from ethanol to give $\mathbf{1 0}$ in $(1.9 \mathrm{~g}) 50 \%$ yield.

Table I. Diindeno[1,2-b:2', $1^{\prime}$-e]pyridines $(\mathbf{9 - 1 1})$. 
Compound 9: Its IR 1710 (CO of indenone), $1420\left(\mathrm{~N}-\mathrm{CH}_{3}\right)$ and $1375 \mathrm{~cm}^{-1}(\mathrm{C}-\mathrm{N}$ stretch. $)$; NMR $\delta 2.50\left(\mathrm{~s}, 3 \mathrm{H}, \mathrm{N}-\mathrm{CH}_{3}\right), 3.65(\mathrm{~s}, 2 \mathrm{H}$, cyclic$\mathrm{CH}_{2}$ ) and $7.3-7.6$ (m, $8 \mathrm{H}$, aromatic protons).

Compound 10: IR 1705 (CO of indenone) and $1360 \mathrm{~cm}^{-1}$ (C-N stretch.); NMR $\delta 2.75$ (s, $2 \mathrm{H}$, benzylic- $\left.\mathrm{CH}_{2}\right), 3.6\left(\mathrm{~s}, 2 \mathrm{H}\right.$, cyclic- $\left.\mathrm{CH}_{2}\right)$ and $7.1-7.9$ (complex pattern, $13 \mathrm{H}$, aromatic protons).

Compound 11: IR broad band at $3490-3420$ $(\mathrm{NH}), 1720$ (CO of indenone) and $1270 \mathrm{~cm}^{-1}$ $(\mathrm{C}-\mathrm{N}$ stretch.); NMR $\delta 2.6(\mathrm{~s}, 1 \mathrm{H}, \mathrm{NH}), 3.55(\mathrm{~s}$, $2 \mathrm{H}$, cyclic- $\left.\mathrm{CH}_{2}\right)$ and $7.2-7.8(\mathrm{~m}, 8 \mathrm{H}$, aromatic protons).

\section{Synthesis of $N, N$-di(3-indenon-1-yl) benzylamine} (12)

A solution of $1(0.04 \mathrm{~mol}, 5.85 \mathrm{~g})$, benzylamine $(0.02 \mathrm{~mol}, 2.19 \mathrm{ml})$ in ethanol $(80 \mathrm{ml})$ and few drops of acetic acid, was kept overnight at room temperature. The separated solid was recrystal- lized from ethanol to give $\mathbf{1 2}$ as greenish crystals, m.p. $87^{\circ} \mathrm{C}$ (decomp.) in ( $\left.4 \mathrm{~g}\right) 55 \%$ yield.

$$
\begin{array}{lrl}
\mathrm{C}_{25} \mathrm{H}_{17} \mathrm{NO}_{2}(363.4) & \\
\text { Calcd } & \mathrm{C} 82.62 & \mathrm{H} 4.70, \\
\text { Found } & \mathrm{C} 82.38 & \mathrm{H} 4.80 .
\end{array}
$$

IR $1710\left(\mathrm{CO}\right.$, indenone) and $1360 \mathrm{~cm}^{-1}(\mathrm{C}-\mathrm{N}$ stretch.).

2-(N-Morpholinobenzyl)-1,3-indandione (13), 2-(N-piperidinobenzyl)-1,3-indandione (14) and $N, N^{\prime}$-bis(1,3-indandione-2-yl-benzyl) piperazine (15)

General procedure: A solution of 2-benzylidene1,3-indandione ( $5 \mathrm{mmol}, 1.17 \mathrm{~g}$ ) and morpholine $(5 \mathrm{mmol}, 0.44 \mathrm{ml})$ or piperidine $(5 \mathrm{mmol}, 0.5 \mathrm{ml})$ or piperazine $(2.5 \mathrm{mmol}, 0.22 \mathrm{~g})$ in $(95 \%)$ ethanol $(30 \mathrm{ml})$ was heated to boiling, kept two days at room temperature, then for another four days in

\begin{tabular}{|c|c|c|c|c|c|c|}
\hline \multirow[t]{2}{*}{ Compound } & \multirow{2}{*}{$\begin{array}{l}\text { M.p. } \\
{\left[{ }^{\circ} \mathrm{C}\right]}\end{array}$} & \multirow{2}{*}{$\begin{array}{l}\text { Yield } \\
{[\%]}\end{array}$} & \multirow{2}{*}{$\begin{array}{l}\text { Formula } \\
\text { (mol. wt.) }\end{array}$} & \multicolumn{2}{|c|}{ Analysis $(\%)$} & \multirow{2}{*}{$\begin{array}{l}\text { Found } \\
\text { (calcd) } \\
\text { N }\end{array}$} \\
\hline & & & & $\mathrm{C}$ & $\mathrm{H}$ & \\
\hline 13 & 280 & 48 & $\begin{array}{l}\mathrm{C}_{20} \mathrm{H}_{19} \mathrm{NO}_{3} \\
(321.4)\end{array}$ & $\begin{array}{c}74.50 \\
(74.75)\end{array}$ & $\begin{array}{c}5.41 \\
(5.96)\end{array}$ & $\begin{array}{c}4.31 \\
(4.36)\end{array}$ \\
\hline 14 & 270 & 55 & $\begin{array}{l}\mathrm{C}_{21} \mathrm{H}_{21} \mathrm{NO}_{2} \\
(319.4)\end{array}$ & $\begin{array}{c}78.35 \\
(78.97)\end{array}$ & $\begin{array}{c}6.38 \\
(6.63)\end{array}$ & $\begin{array}{c}4.28 \\
(4.39)\end{array}$ \\
\hline 15 & 202 & 60 & $\begin{array}{l}\mathrm{C}_{36} \mathrm{H}_{30} \mathrm{~N}_{2} \mathrm{O}_{4} \\
(554.6)\end{array}$ & $\begin{array}{l}77.58 \\
(77.96)\end{array}$ & $\begin{array}{c}5.20 \\
(5.45)\end{array}$ & $\begin{array}{c}5.01 \\
(5.05)\end{array}$ \\
\hline
\end{tabular}
ice-chest. The products obtained were recrystallized from ethanol as yellow crystals ( $c f$. Table II).

Table II. Mannich bases 13-15.

[1] R. S. Varna and W. L. Nobles, J. Pharm. Sci. 55, 1451 (1966).

[2] A. Arens, V. Zelmere, E. Shofro, and S. Germane, USSR, Pat. 493, 465 (1976); C. A. 84, 89877 w (1976).

[3] The Merk Index of Chemicals and Drugs, 9th ed., item No. 6752, Merk and Co., Inc. Rahway, N.J. U.S.A. (1976).

[4] F. Amer, E. M. Afsah, and H. Etman, Z. Naturforsch. 34 b, 867 (1979).

[5] E. M. Afsah, H. M. Hassan, S. A. Elagizy, and M. T. Zimaity, J. Prakt. Chem. 326, 841 (1984).

[6] M. Hammouda, W. S. Hamama, and E. M. Afsah, Z. Naturforsch. 42 b, 94 (1987).
[7] M. Hammouda, W. S. Hamama, E. M. Kandeel, and E. M. Afsah, Pharmazie 43, 529 (1988).

[8] W. S. Hamama, M. Hammouda, and E. M. Afsah, Z. Naturforsch. 43b, 897 (1988)

[9] D. Radulescu, V. Georgescu, Bull. Soc. Chim. Fr. 37, 1069 (1925); C. A. 19, 3486 (1925).

[10] Z. Vorlander, Anal. Chem. 77, 241 (1929).

[11] E. M. Afsah, A. A. Sarhan, and M. R. Ibraham, J. Prakt. Chem. 326, 683 (1984).

[12] J. Geeenhil, J. Chem. Soc. (C) 1971, 2699.

[13] M. V. Ionescu, Bull. Soc. Chim. Fr. 47, 210 (1930). 